\title{
Coupling of Solid-State and Electromagnetic Equations for Simulation of Wireless Packaged Geometries
}

\author{
B. McGarvey*, M. Tentzeris \\ * The Georgia Institute of Technology, School of Electrical and Computer Engineering, \\ Atlanta, GA 30332-0250, U.S.A., bmcgarvey@ece.gatech.edu \\ The Georgia Institute of Technology, School of Electrical and Computer Engineering, \\ Atlanta, GA 30332-0250, U.S.A., etentze@ece.gatech.edu
}

Abstract: The balanced semiconductor solid-state equations as derived in [1] are efficiently coupled to Yee's FDTD model for Maxwell's electromagnetic equations. The resulting algorithm has capabilities of accurate and effective time-domain simulation of both passive and active devices, which can be used in wireless devices. A novel method that correlates the CFL requirements of the electromagnetic and the device simulator is presented and its application further enhances the numerical stability of the new numerical technique even for sub-micron devices. The discretization problems of a fully adaptive implementation are also discussed in detail.

\section{Introduction}

The accurate design and optimization of state-of-the-art wireless devices requires a simulator that can model both active and passive devices. Various solid-state models exist for these types of devices [1] and have been previously integrated with electromagnetic simulators (EMS), such as FDTD [2, 3, 4]. However, most of the prior implantations of this coupling have been limited in application due to the fact that the application of the CFL conditions [1] and stability requirements for the two models yields disparate time steps often with several orders of magnitude difference [2, 3]. The active device simulator (DS) has the smallest time and space stepping. The use of these values for the whole simulator makes the computational and memory requirements prohibitive for modeling an active device with integrated passives in a package. One alternative approach includes the lumped-element model of the device, but that method requires $a$ priori knowledge of the device performance [5]. The novel approach proposed in this paper involves the development of a fully adaptive scheme in time- and space-domain that allows for the simultaneous timedomain simulation of the devices using different time and space discretization steps for the two sets of equations depending on the local details and the field variations.

\section{Balanced Carrier Solid-State Equations}

Accurate Prediction models for packaged wireless circuits requires the development of a global simulator including both electromagnetic and solid-state equations. Most of these approaches involve the simultaneous solution of the Maxwell's equations and of the balanced equation model [1, 4] that can be written in terms of the majority carrier density $n$, velocity $\vec{v}_{d}$, energy $w$, and electric potential $\varphi$ :

$$
\begin{gathered}
\frac{\partial n}{\partial t}=\nabla \cdot\left(n \vec{v}_{d}\right) \\
\frac{\partial\left(\vec{v}_{d}\right)}{\partial t}=-\frac{\vec{v}_{d}}{m^{*}} \nabla \cdot\left(m^{*} \vec{v}_{d}\right)+\frac{q \vec{E}}{m^{*}}-\frac{2}{3 n m^{*}} \nabla\left(n w-\frac{1}{2} m^{*} n \vec{v}_{d}^{2}\right) \\
\frac{\partial w}{\partial t}=-\vec{v}_{d} \nabla \cdot w-\frac{2}{3 n} \nabla \cdot\left[\left(n \vec{v}_{d}-\frac{\kappa}{k_{B}} \nabla\right)\left(w-\frac{m^{*} \vec{v}_{d}^{2}}{2}\right)\right]+q \vec{E} \cdot \vec{v}_{d} \\
\nabla^{2} \varphi=-\frac{q}{\varepsilon}\left(N_{D}-n_{i}\right)
\end{gathered}
$$

$\begin{array}{ll}n: & \text { Carrier Concentration } \\ \vec{v}_{d}: & \text { Carrier Velocity } \\ m^{*}: & \text { Carrier Effective Mass } \\ q: & \text { Electron Charge }\end{array}$ $w:$ Carrier Energy

$\varphi$ : Electric Potential

$\vec{E}$ : Electric Field

$T$ : Temp ( ${ }^{\circ}$ Kelvin)
$N_{D}$ : Majority Carrier Density

$n_{i}$ : Intrinsic Carrier Concentration

$k_{B}$ : Boltzmann's Constant

$k$ : Thermal Conductivity 
The discretization of the Equations (1)-(4) is accomplished using forward Euler for the time derivative and central differencing method for the spatial derivatives. As an example, Eq. (1) can be written as

$$
\frac{\Delta n_{i}^{k+1}-\Delta n_{i}^{k}}{\Delta t_{D S}}=-\left[\left(\frac{\vec{v}_{d x_{i+1 / 2}}^{k}+\vec{v}_{d x_{i-1 / 2}}^{k}}{2}\right)\left(\frac{\Delta n_{i+1}^{k}-\Delta n_{i}^{k}}{\Delta x}\right)-\left(n_{i}^{k}\right)\left(\frac{\vec{v}_{d x_{i+1 / 2}}^{k}-\vec{v}_{d x_{i-1 / 2}^{k}}}{\Delta x}\right)\right],
$$

where $u(n \Delta t, i \Delta x)=u_{i}^{n}$ is used. The discretized equations are imposed on the grid proposed in $[2,3]$ that places the scalar quantities $(n, w)$ at the nodal points and the vector quantities $\left(\vec{v}_{d}, \vec{E}\right)$ at the mid-cell points as illustrated in Fig. 1.

\section{Stability of the Novel Coupled Algorithm}

The cell size of the EMS model is limited by the maximum frequency, $\mathrm{f}_{\max }$, of the excitation for a basic Yee FDTD model and is usually smaller than one-tenth the wavelength. In the balanced solid-state system, the Debye Length limits the spatial step to maintain acceptable numerical accuracy, and is also usually less than one-tenth the Debye Length. Specifically, the spatial discretization of the solid-state equations involves determining the Debye Length of the semiconductor, which can be calculated using Eq. (8), and is only a function of the doping level and other material characteristics $[6,8]$. The time step is a function of the average carrier velocity and the spatial step to comply with the CFL condition (7) for stability and minimizing numerical dispersion.

$$
\begin{array}{ll}
v_{p}=\text { Phase velocity in EMS } ; & \text { CFL condition for the EMS is } v_{p}<\frac{\Delta x_{E M S}}{\Delta t_{E M S}} \\
v_{d}=\text { Average carrier velocity in DS; } & \text { CFL condition for the DS is } v_{d}<\frac{\Delta x_{D S}}{\Delta t_{D S}} \\
\varepsilon_{r}=\text { Dielectric Constant } & \text { Debye Length }=L_{D}=\sqrt{\frac{\varepsilon_{r} \varepsilon_{o} k_{B} T}{q^{2} N_{D}}}
\end{array}
$$

Therefore, the device simulator space step is an independent variable and a prime candidate for adaptive stepping. Fig. 2 shows a possible implementation of the adaptive gridding with the cell size as a function of the doping level. The cell size is at a minimum in the heavily doped section, and increases as the doping decreases, until it reaches a maximum in the intrinsic region. The implementation of an adaptive gridding scheme helps to reduce the number of cells required for a stable and accurate simulation. Memory and computation savings for the device simulator varies directly with respect to the doping profile.

\section{Space and Time Discretization}

Discretizing an example device highlights the main difficulties in coupling the two systems: time and space step disparity. A sample Silicon device has the following properties: heavily doped $10^{18} \mathrm{~cm}^{-3}$, large applied electric field, and an EMS excitation with $\mathrm{f}_{\max }=10 \mathrm{GHz}$. The DS cell size is $10^{-8} \mathrm{~m}$ as shown in Fig 3 , and the EMS space step is approximately $10^{-4} \mathrm{~m}$ as shown in Fig 4 . The spatial discrepancy can be managed by limiting the size of the devices simulated. For the given device, the DS time step is $10^{-14} \mathrm{~s}$, as presented in Fig 5, and the EMS time step is $10^{-11} \mathrm{~s}$, for a difference in magnitude of $10^{3}$. Fig 5 shows the relationship of $\Delta t_{D S}$ to the CFL (7) and drift velocity (9). The cell size $\left(\Delta x_{D S}\right)$ is determined by the Debye Length (8), and is primarily dependent on the carrier concentration. The time step is dependent upon the drift velocity and varies inversely to the applied electric field, as seen in Fig 5. The figure presents a set of parametric curves showing relation ship of the electric field varying from $10^{-3} \mathrm{~V} / \mathrm{m}, 10^{-2} \mathrm{~V} / \mathrm{m}$, to $10^{7} \mathrm{~V} / \mathrm{m}$ and the space and time discretization.

The time step imbalance poses a significant problem that must be solved. Selecting the smallest time step of the systems would cause an unreasonable growth in the execution time and greater numerical dispersion. Choosing independent time steps for the systems makes the increase in required computational requirements manageable and keeps the small time steps localized to the device, but makes effective coupling difficult. The imbalance causes the change in the electromagnetic excitation for the device to be extremely localized in time and induces a shock into the system. This shock is non-physical and must be smoothed. This is accomplished by dividing the large electromagnetic time step into many smaller time 
steps appropriate for the semiconductor model effectively smoothing the step excitation to a continuous function, as illustrated in Fig 6.

Calculating the semiconductor time step, with the small signal applied, becomes slightly more complex. The system is simulating both the DC biasing and the small signal effects on the device. If the CFL condition is violated, the results are erroneous. The applied voltage becomes the sum of $V_{A C}+V_{D C}=V_{\text {Total }}$. This result is applied to Eq. (7) and (9) to calculate the new drift velocity and time step.

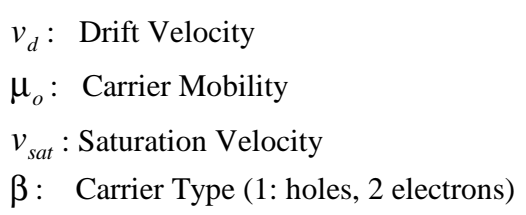

$$
v_{d}=\frac{\mu_{o} \vec{E}}{\left[1+\left(\frac{\mu_{o} \vec{E}}{v_{s a t}}\right)^{\beta}\right]^{\frac{1}{\beta}}}
$$

\section{Conclusion}

The novel approach proposed in this paper involves the development of a fully adaptive scheme in the time- and space-domains. This method provides for a time-domain simulation of active and passive devices, using two systems of equations that are dependent on the local details and field variations.

\section{Acknowledgements}

The authors would like to acknowledge the support of the NSF Packaging Research Center at Georgia Tech, the Yamacraw Design Center and the NSF Career Award.

\section{References}

[1] K. Tomizawa, "Numerical Simulation of Submicron Semiconductor Devices", Norwood, MA: Artech House Inc., 1993.

[2] P. Caimpolini, L. Roselli, and G. Stopponi "Integrated FDTD and Solid-State Simulation," IEEE and Guided Wave Letters, Vol 6, No. 11, pp. 419-421, November 1996.

[3] M. Alsunaidi, A. Imtiaz, S. M. El-Ghazaly Electromagnetic, "Wave Effects on Microwave Transistors Using a Full-Wave Time-Domain Model", IEEE Transactions on Microwave Theory and Techniques, Vol 44, No. 6, pp. 799-808, June 1996.

[4] A. Taflove, Computational Electrodynamics: the finite difference time domain method, Norwood, MA: Artech House Inc, 1995.

[5] L. Roselli, E. Tentzeris, and L.P.B.Katehi, "Nonlinear Circuit Characterization Using a Multiresolution Time Domain Technique", Proc. of the 1998 MTT-S Conference, pp.1387-1390, Baltimore, MD, June 1998.

[6] S. M. Sze, "Physics of Semiconductor Devices", New York, NY: John Wikey \& Sons, Inc. 1981.

[7] R. Pierret, "Semiconductor Device Fundamentals", New York, NY: Addison-Wesley Publishing Company Inc., 1996.

[8] A. Imtiaz, S. M. El-Ghazaly, "Global modeling of millimeter-Wave Circuits: Electromagnetic Simulation of Amplifiers", IEEE Transactions on Microwave Theory and Techniques, Vol. 45 No. 12, pp. 2208-2216, December 1997.

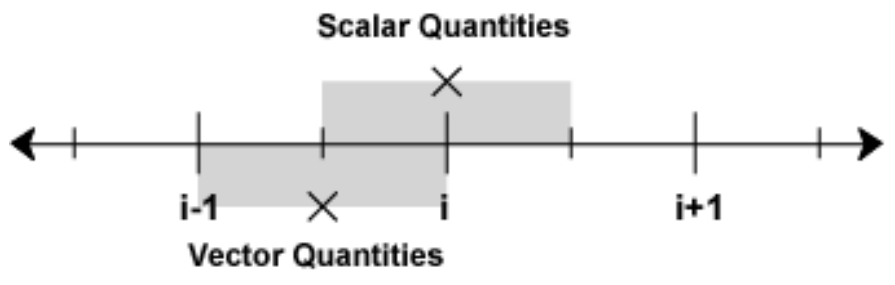

Figure 1. Grid Setup for Device Simulator 




Figure 2. Adaptive gridding for cell size vs. doping levels

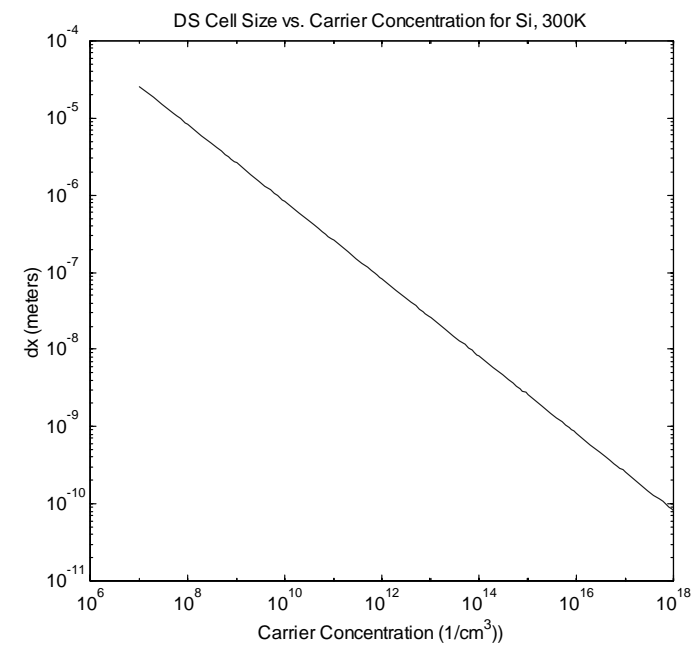

Figure 3. DS Cell Size vs. Carrier Concentration

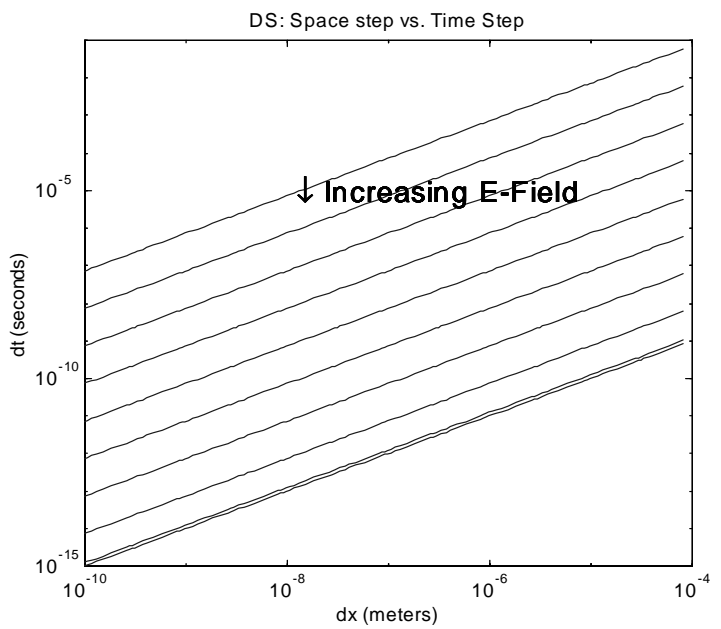

Figure 5. DS: Relation of E-field, Space Step and Time Step

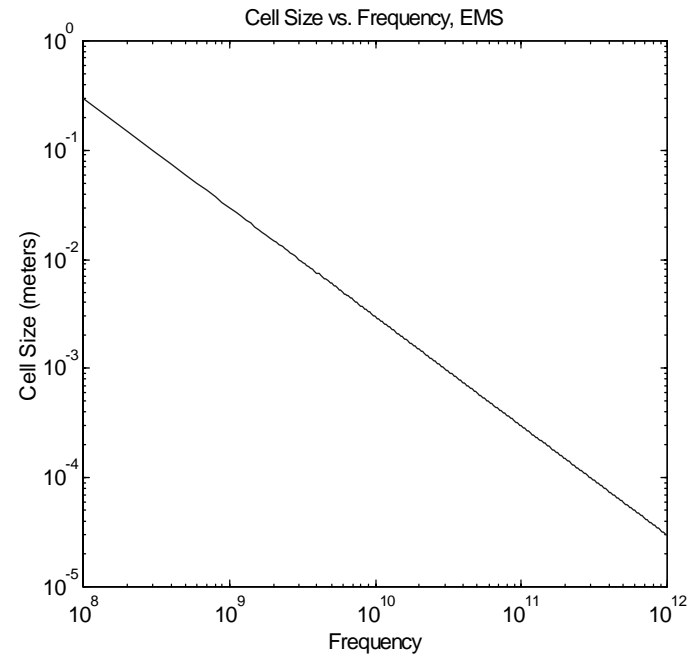

Figure 4. EMS: Cell Size vs. Frequency



Figure 6. EMS to DS Excitation Smoothing 